\title{
ON THE DARBOUX INTEGRABILITY OF BLASIUS AND FALKNER-SKAN EQUATION
}

\author{
JAUME LLIBRE ${ }^{1}$, AND CLAUDIA VALLS $^{2}$
}

\begin{abstract}
We study the Darboux integrability of the celebrated FalknerSkan equation $f^{\prime \prime \prime}+f f^{\prime \prime}+\lambda\left(1-f^{\prime 2}\right)=0$, where $\lambda$ is a parameter. When $\lambda=0$ this equation is known as Blasius equation. We show that both differential systems have no first integrals of Darboux type. Additionally we compute all the Darboux polynomials and all the exponential factors of these differential equations.
\end{abstract}

\section{Introduction and Statement of the main RESUlts}

The Falkner-Skan equation is

$$
f^{\prime \prime \prime}+f f^{\prime \prime}+\lambda\left(1-f^{\prime 2}\right)=0
$$

where $\lambda \in \mathbb{R}$ is a parameter. This equation was first derived in [6] as a model of the steady two-dimensional flow of a slightly viscous incompressible fluid past a wedge. The special case $\lambda=0$, in which the wedge reduces to a flat plate, is called Blasius equation and was considered for a first time in [2].

Both equations are the subject of an extensive literature. For the derivation of this equation see [1]. For the existence and uniqueness of the solutions

2000 Mathematics Subject Classification. 34C05, 34A34, 34C14.

Key words and phrases. Falkner-Skan equation, Blasius equation, integrability, Darboux polynomials. 
see, for example, [16], [19], [5], [15], [3] and [10] and references therein. Recently there has been also a renewed interest in the mathematical aspects of the Falkner-Skan equation. The dynamic features of this equation such as the existence of oscillating and periodic orbits have been studied in [7], [8], [9] and [13]; and for more recent works on the bifurcations in this equation see [11], [18] and [17].

In MathSciNet appears in this moment 214 articles related with the Falkner-Skan equation, but any of these papers analyze the integrability or non-integrability of this equation. In this work we are interested in the Darboux integrability of Blasius and Falkner-Skan equation. Before we state our main result (Theorem 1) we need to introduce some definitions and auxiliary results.

We can express (1) as a system of differential equations

$$
\dot{x}=y, \quad \dot{y}=z \quad \dot{z}=-x z-\lambda\left(1-y^{2}\right),
$$

and the associated vector field is

$$
\mathcal{X}=y \frac{\partial}{\partial x}+z \frac{\partial}{\partial y}+\left[-x z-\lambda\left(1-y^{2}\right)\right] \frac{\partial}{\partial z}
$$

Let $U \subset \mathbb{R}^{3}$ be an open subset. We say that the non-constant function $H: U \rightarrow \mathbb{R}$ is the first integral of the polynomial vector field (3) on $U$ associated to system (2), if $H(x(t), y(t), z(t))=$ constant for all values of $t$ for which the solution $(x(t), y(t), z(t))$ of $\mathcal{X}$ is defined on $U$. Clearly $H$ 
ON THE DARBOUX INTEGRABILITY OF BLASIUS AND FALKNER-SKAN EQUATIOß is a first integral of $\mathcal{X}$ on $U$ if and only if $\mathcal{X} H=0$ on $U$. When $H$ is a polynomial we say that $H$ is a polynomial first integral.

For proving our main results concerning the existence of first integrals of Darboux type we shall use the invariant algebraic surfaces of system (2). This is the basis of the Darboux theory of integrability. The Darboux theory of integrability works for real or complex polynomial ordinary differential equations. The study of complex invariant algebraic curves is necessary for obtaining all the real first integrals of a real polynomial differential equation, for more details see [12].

Let $h=h(x, y, z) \in \mathbb{C}[x, y, z]$ be a non-constant polynomial. We say that $h=0$ is an invariant algebraic surface of the vector field $\mathcal{X}$ in (3) if it satisfies $\mathcal{X} h=K h$, for some polynomial $K=K(x, y, z) \in \mathbb{C}[x, y, z]$, called the cofactor of $h$. Note that $K$ has degree at most 1 . The polynomial $h$ is called a Darboux polynomial, and we also say that $K$ is the cofactor of the Darboux polynomial $h$. We note that a Darboux polynomial with zero cofactor is a polynomial first integral.

Let $g, h \in \mathbb{C}[x, y, z]$ be coprime. We say that a non-constant function $E=e^{h / g}$ is an exponential factor of the vector field $\mathcal{X}$ given in (3) if it satisfies $\mathcal{X} E=L E$, for some polynomial $L=L(x, y, z) \in \mathbb{C}[x, y, z]$, called the cofactor of $E$ and having degree at most 1. This relation is equivalent to

$$
y \frac{\partial(g / h)}{\partial x}+z \frac{\partial(g / h)}{\partial y}+\left[-x z-\lambda\left(1-y^{2}\right)\right] \frac{\partial(g / h)}{\partial z}=K .
$$


For a geometrical and algebraic meaning of the exponential factors see [4].

A first integral $G$ of system (2) is called of Darboux type if it is of the form

$$
G=f_{1}^{\lambda_{1}} \cdots f_{p}^{\lambda_{p}} E_{1}^{\mu_{1}} \cdots E_{q}^{\mu_{q}}
$$

where $f_{1}, \ldots, f_{p}$ are Darboux polynomials, $E_{1}, \ldots, E_{q}$ are exponential factors and $\lambda_{j}, \mu_{k} \in \mathbb{C}$ for $j=1, \ldots, p, k=1, \ldots, q$. For more information on the Darboux theory of integrability see, for instance, $[12,14]$ and the references quoted there.

The main result of this paper is the following:

Theorem 1. For the Falkner-Skan and Blasius system the following statements hold.

(a) Both systems have no polynomial first integrals.

(b) The unique irreducible Darboux polynomial with nonzero cofactor of the Blasius system is $z$; the unique Darboux polynomial of the Falkner-Skan system is $1-y^{2}+2 x z$ if $\lambda=1 / 2$.

(c) The unique exponential factors of both systems are $e^{x}$ and $e^{y}$, except if $\lambda=-1$ then we have the additional exponential factor $e^{z+x y}$.

(d) Both systems have no first integrals of Darboux type.

Theorem 1 is proved in the next section. 
ON THE DARBOUX INTEGRABILITY OF BLASIUS AND FALKNER-SKAN EQUATION

\section{Proof of Theorem 1}

We separate the proof of Theorem 1 in four propositions, one for every statement.

Proposition 2. System (2) has no polynomial first integrals.

Proof. Let $h$ be a polynomial first integral of system (2). Then it satisfies

$$
y \frac{\partial h}{\partial x}+z \frac{\partial h}{\partial y}+\left[-x z-\lambda\left(1-y^{2}\right)\right] \frac{\partial h}{\partial z}=0 .
$$

Without loss of generality we can write $h=\sum_{j=1}^{n} h_{j}(x, y, z)$, where each $h_{j}=h_{j}(x, y, z)$ is a homogeneous polynomial of degree $j$, and $h_{n} \neq 0$.

Computing the terms of degree $n+1$ in (5) we get

$$
\left[-x z+\lambda y^{2}\right] \frac{\partial h_{n}}{\partial z}=0
$$

Therefore $h_{n}=h_{n}(x, y)$.

Computing the terms of degree $n$ in (5) we get that

$$
y \frac{\partial h_{n}}{\partial x}+z \frac{\partial h_{n}}{\partial y}+\left[-x z+\lambda y^{2}\right] \frac{\partial h_{n-1}}{\partial z}=0,
$$

that is

$$
h_{n-1}=g_{n-1}(x, y)+\frac{z}{x} \frac{\partial h_{n}}{\partial y}+\frac{y}{x^{2}} \log \left(x z-\lambda y^{2}\right)\left[\lambda y \frac{\partial h_{n}}{\partial y}+x \frac{\partial h_{n}}{\partial x}\right]
$$


where $g_{n-1}(x, y)$ is a function in the variables $x$ and $y$. Since $h_{n-1}$ is a polynomial, we have

$$
\lambda y \frac{\partial h_{n}}{\partial y}+x \frac{\partial h_{n}}{\partial x}=0
$$

Therefore $h_{n}=h_{n}\left(x^{-\lambda} y\right)$. Since $h_{n} \neq 0$ is a homogeneous polynomial of degree $n \geq 1$, we have $\lambda=-p / q$ with $p$ and $q$ integers such that $p \geq 0$, $q \geq 1, p+q=n$ and $h_{n}=\alpha_{n} x^{p} y^{q}$, where $\alpha_{n} \in \mathbb{C} \backslash\{0\}$. Of course in the case of the Blasius system $p=0$. In short we get that $h_{n}=\alpha_{n} x^{p} y^{q}$.

Now we have that

$$
h_{n-1}(x, y, z)=\alpha_{n} q x^{p-1} y^{q-1} z+g_{n-1}(x, y) .
$$

So for the Blasius system $(p=0)$ we have a contradiction with the fact that $h_{n-1}(x, y, z)$ is a homogeneous polynomial of degree $n-1$. Thus the proposition is proved for the Blasius system. In what follows we assume that $p \geq 1$, i.e. we restrict our attention to the Falkner-Skan system.

Computing the terms of degree $n-1$ in (5) we get that

$$
y \frac{\partial h_{n-1}}{\partial x}+z \frac{\partial h_{n-1}}{\partial y}+\left[-x z+\lambda y^{2}\right] \frac{\partial h_{n-2}}{\partial z}=0
$$

that is

$$
\begin{aligned}
h_{n-2}= & g_{n-2}(x, y)+\frac{1}{2} \alpha_{n}\left[2(p-q) y^{2}+(q-1) q x z\right] x^{p-3} y^{q-2} z+ \\
& \frac{z}{x} \frac{\partial g_{n-1}}{\partial y}+\frac{y}{q x^{4}} \log \left(p y^{2}+q x z\right) A,
\end{aligned}
$$


where

$$
A=-\alpha_{n} p(p-q) x^{p} y^{q+1}+q x^{3} \frac{\partial g_{n-1}}{\partial x}-p x^{2} y \frac{\partial g_{n-1}}{\partial y} .
$$

Since $h_{n-2}$ is a homogeneous polynomial of degree $n-2$ we have that $A=0$.

Solving this linear partial differential equation we get that

$$
g_{n-1}(x, y, z)=-\frac{1}{p+2 q} \alpha_{n} p(p-q) x^{p-2} y^{q+1}+G\left(x^{p / q} y\right) .
$$

Since $p+q=n$ and $g_{n-1}$ is a homogeneous polynomial of degree $n-1$ we have that

$$
g_{n-1}(x, y, z)=-\frac{1}{p+2 q} \alpha_{n} p(p-q) x^{p-2} y^{q+1}
$$

Therefore

$$
\begin{aligned}
h_{n-1}= & \alpha_{n} q x^{p-1} y^{q-1} z-\frac{1}{p+2 q} \alpha_{n} p(p-q) x^{p-2} y^{q+1}, \\
h_{n-2}= & g_{n-2}(x, y)+\frac{1}{2} \alpha_{n}\left[2(p-q) y^{2}+(q-1) q x z\right] x^{p-3} y^{q-2} z- \\
& \frac{1}{p+2 q} \alpha_{n} p(p-q)(q+1) x^{p-3} y^{q} z .
\end{aligned}
$$

Note that $h_{n-1}$ is a polynomial if $p \geq 1$ and $q \geq 1$, so $n \geq 2$; and that $h_{n-2}$ is a polynomial if $p \geq 3$ and $q \geq 2$, so $n \geq 5$.

Working in a similar way with the terms of degree $n-2$ in (5) we get that

$$
\begin{aligned}
g_{n-2}= & \frac{\alpha_{n} x^{p-4} y^{q-2}}{2 q(p+2 q)^{2}}\left[-q^{2}(p+2 q)^{2} x^{4}+\right. \\
& \left.p\left(p^{3}(q-1)-6 q^{3}+p q^{2}(8+q)-p^{2} q(1+2 q)\right) y^{4}\right]
\end{aligned}
$$


and

$$
\begin{aligned}
h_{n-3}= & \frac{1}{6(p+2 q)^{2}}\left[6(p+2 q)^{2} g_{n-3}(x, y)+\alpha_{n} x^{p-5} y^{q-3} z\left(3(2-q) q(p+2 q)^{2} x^{4}+\right.\right. \\
& 3(p-4)(p-q)\left(6 q^{2}-p^{2}-2 p q+p^{2} q-p q^{2}\right) y^{4}+ \\
& 3(-p+q)(p+2 q)\left(p+2 q-p q-4 q^{2}+p q^{2}\right) x y^{2} z+ \\
& \left.\left.(q-2)(q-1) q(p+2 q)^{2} x^{2} z^{2}\right)\right]
\end{aligned}
$$

where $g_{n-3}$ is an arbitrary polynomial of degree $n-3$. Since $h_{n-3}$ is a polynomial we must have that $p \geq 5$ and $q \geq 3$, so $n \geq 8$.

In short if there exists a polynomial first integral of degree $n$, then $h_{n-s}$ is a polynomial if $p \geq 2 s-1$ and $q \geq s$, so $n \geq 3 s-1$. Thus for $s=n-1$ we get that $h_{1}$ is a polynomial if $n \geq 3(n-1)-1=3 n-4$, i.e. if and only if $4 \geq 2 n$, or equivalently if $n=1,2$. But direct computations shows that system (2) has no polynomial first integrals of degree 1 and 2. This completes the proof of the proposition.

The following result on the Darboux polynomials is well known, see for instance [12]. This result shows that it is sufficient to know the irreducible Darboux polynomials.

Proposition 3. Let $f$ be a polynomial and $f=\prod_{j=1}^{s} f_{j}^{\alpha_{j}}$ its decomposition into irreducible factors in $\mathbb{C}[x, y, z]$. Then $f$ is a Darboux polynomial if and only if all the $f_{j}$ are Darboux polynomials. Moreover, if $K$ and $K_{j}$ are the cofactors of $f$ and $f_{j}$, then $K=\sum_{j=1}^{s} \alpha_{j} K_{j}$. 
Proposition 4. The following statements hold.

(a) If $\lambda=0$ then the unique irreducible Darboux polynomial with nonzero cofactor of system (2) is $z$.

(b) If $\lambda \neq 0$ then system (2) has no Darboux polynomials, except if $\lambda=1 / 2$ then $x z-y^{2} / 2+1 / 2$ is the unique irreducible Darboux polynomial.

Proof. Let $h$ be an irreducible Darboux polynomial of system (2) with a nonzero cofactor $K$. Then $K=\alpha_{0}+\alpha_{1} x+\alpha_{2} y+\alpha_{3} z$, with $\alpha_{i} \in \mathbb{C}$ for $i=0,1,2,3$ not all zero.

It is easy to see by direct computations that if $h$ has degree 1 , then $h=z$ and $\lambda=0$.

Now we assume that $h$ has degree greater $\geq 2$. Then it satisfies

(6) $y \frac{\partial h}{\partial x}+z \frac{\partial h}{\partial y}-\left[x z+\lambda\left(1-y^{2}\right)\right] \frac{\partial h}{\partial y}=\left(\alpha_{0}+\alpha_{1} x+\alpha_{2} y+\alpha_{3} z\right) h$.

We write $h=\sum_{j=0}^{n} h_{j}(x, y, z)$, where each $h_{j}=h_{j}(x, y, z)$ is a homogeneous polynomial of degree $j$ and $h_{n} \neq 0$.

Computing the terms of degree $n+1$ in (6) we get that

$$
\left(\lambda y^{2}-x z\right) \frac{\partial h_{n}}{\partial z}=\left(\alpha_{1} x+\alpha_{2} y+\alpha_{3} z\right) h_{n}
$$

Solving this linear differential equation we obtain

$$
h_{n}=g_{n}(x, y) e^{-\alpha_{3} z / x}\left(x z-\lambda y^{2}\right)^{-\alpha_{1}-y\left(\alpha_{2} x+\alpha_{3} \lambda y\right) / x^{2}},
$$


where $g_{n}(x, y)$ is a function in the variables $x$ and $y$. Since $h_{n}$ is a homogeneous polynomial of degree $n$ we must have $\alpha_{3}=\alpha_{2}=0$ and $\alpha_{1}=-m$ for some integer $m$ with $0 \leq m \leq n / 2$ and $g_{n} \neq 0$. That is $h_{n}=$ $g_{n}(x, y)\left(x z-\lambda y^{2}\right)^{m}$ with $g_{n}$ a homogeneous polynomial of degree $n-2 m$.

Assume $\lambda=0$. Then, using that $z=0$ is invariant for system (2), we can write $h=\bar{h}(x, y)+z h_{1}(x, y, z)$. Then $\bar{h}$ satisfies (6) restricted to $z=0$ (and with $\alpha_{1}=-m, \alpha_{2}=\alpha_{3}=0$ ). Then $\bar{h} \neq 0$ (otherwise $h$ would be reducible) satisfies

$$
y \frac{\partial \bar{h}}{\partial x}=\left(\alpha_{0}-m x\right) \bar{h}
$$

Solving this linear differential equation we get that

$$
\bar{h}=e^{-\alpha_{0} x / y-m x^{2} /(2 y)} \bar{g}(y),
$$

where $\bar{g}(y)$ is a function in the variable $y$. Using that $\bar{h}$ is a polynomial we have $\alpha_{0}=m=0$. But then $K=0$, a contradiction with the fact that $h$ is a Darboux polynomial with nonzero cofactor. This concludes the proof of statement (a).

Now assume that $\lambda=1 / 2$. Then, it is easy to check that if $h$ has degree 2 , then $h=T=x z-y^{2}-1 / 2=0$ is invariant for system (2). Therefore we assume that the degree of $h$ is at least 3 . Then if we rewrite system (2) in the variables $(x, y, T)$ with $z=\left(T+y^{2} / 2-1 / 2\right) / x$ we obtain the system

$$
\dot{x}=y, \quad \dot{y}=\frac{T+y^{2} / 2-1 / 2}{x}, \quad \dot{T}=-x T .
$$


ON THE DARBOUX INTEGRABILITY OF BLASIUS AND FALKNER-SKAN EQUATIOIN Then we have that $h^{*}(x, y, T)=h(x, y, z)$ and it can be written as $h^{*}(x, y, T)=$ $\tilde{h}(x, y)+T \tilde{h}_{1}(x, y, T)$, where $\tilde{h}(x, y)$ is a polynomial in the variable $y$ (not in the variable $x$ ) and $\tilde{h}_{1}$ is a polynomial in the variables $x$ and $T$. Now equation (6) written in the variables $x, y$ and $T$, and restricted to $T=0$ becomes

$$
y \frac{\partial \tilde{h}}{\partial x}+\frac{y^{2}-1}{2 x} \frac{\partial \tilde{h}}{\partial y}=\left(\alpha_{0}-m x\right) \tilde{h}
$$

Solving this partial differential equation we get that

$$
\tilde{h}=e^{2 x y\left[m x\left(y^{2}-3\right)-3 \alpha_{0}\left(y^{2}-1\right)\right] /\left(3\left(y^{2}-1\right)^{2}\right)} \tilde{g}\left[\frac{1}{2} \log \left(\frac{y^{2}-1}{x}\right)\right],
$$

where $\tilde{g}(\cdot)$ is a function in its variable. Using that $\tilde{h}$ is a polynomial in the variable $y$ we have $\alpha_{0}=m=0$. But then $K=0$, a contradiction with the fact that $h$ is a Darboux polynomial with nonzero cofactor. This concludes the proof of statement (b) when $\lambda=1 / 2$.

Suppose $h$ is an irreducible Darboux polynomial of system (2) with $\lambda \notin$ $\{0,1 / 2\}$ and with nonzero cofactor $K$. Then $K=\alpha_{0}+\alpha_{1} x+\alpha_{2} y+\alpha_{3} z$, with $\alpha_{i} \in \mathbb{C}$ for $i=0,1,2,3$ not all zero.

We shall assume that the polynomial $h$ has degree $\geq 2$, because it is easy to see by direct computations that system (2) with $\lambda \notin\{0,1 / 2\}$ has no Darboux polynomials of degree 1 .

Clearly a Darboux polynomial $h$ satisfies

$$
y \frac{\partial h}{\partial x}+z \frac{\partial h}{\partial y}+\left[-x z-\lambda\left(1-y^{2}\right)\right] \frac{\partial h}{\partial z}=\left(\alpha_{0}+\alpha_{1} x+\alpha_{2} y+\alpha_{3} z\right) h .
$$


We write $h$ as $h=\sum_{j=0}^{n} h_{j}(x, y, z)$, where each $h_{j}=h_{j}(x, y, z)$ is a homogeneous polynomial of degree $j$ and $h_{n} \neq 0$ with $n>2$.

Computing the terms of degree $n+1$ in (7) we get that

$$
\left[-x z+\lambda y^{2}\right] \frac{\partial h_{n}}{\partial z}=\left(\alpha_{1} x+\alpha_{2} y+\alpha_{3} z\right) h_{n}
$$

Solving this linear differential equation we obtain

$$
h_{n}=g_{n}(x, y) e^{-\alpha_{3} z / x}\left(x z-\lambda y^{2}\right)^{-\alpha_{3} \lambda y^{2} / x^{2}-\alpha_{2} y / x-\alpha_{1}},
$$

where $g_{n}=g_{n}(x, y)$ is a function in the variables $x, y$. Since $h_{n}$ is a homogeneous polynomial of degree $n$ we must have $\alpha_{3}=\alpha_{2}=0$ and $\alpha_{1}=-m$ for some integer $m$ with $0 \leq 2 m \leq n$ and $g_{n}$ is a homogeneous polynomial of degree $n-2 m$ in $x$ and $y$. Then

$$
h_{n}=\left(x z-\lambda y^{2}\right)^{m} g_{n},
$$

for some integer $m$ such that $0 \leq 2 m \leq n$.

Computing the terms of degree $n$ in (7) we get that

$$
y \frac{\partial h_{n}}{\partial x}+z \frac{\partial h_{n}}{\partial y}+\left[-x z+\lambda y^{2}\right] \frac{\partial h_{n-1}}{\partial z}-\alpha_{0} h_{n}+m x h_{n-1}=0 .
$$


Solving it we obtain

$$
\begin{aligned}
h_{n-1}= & \left(x z-\lambda y^{2}\right)^{m}\left[g_{n-1}(x, y)+\frac{1}{x^{2}}\left(\frac{m \lambda(2 \lambda-1) y^{3} g_{n}}{x z-y^{2} \lambda}+\left(x z-\lambda y^{2}\right) \frac{\partial g_{n}}{\partial y}+\right.\right. \\
& \left.\left.\log \left(x z-\lambda y^{2}\right)\left[\left(m y(1-2 \lambda)-\alpha_{0} x\right) g_{n}+\lambda y^{2} \frac{\partial g_{n}}{\partial y}+x y \frac{\partial g_{n}}{\partial x}\right]\right)\right],
\end{aligned}
$$

where $g_{n-1}=g_{n-1}(x, y)$ is a function in the variables $x, y$. Since $h_{n-1}$ is a homogeneous polynomial of degree $n-1$ we have that

$$
\left[m y(1-2 \lambda)-\alpha_{0} x\right] g_{n}+y\left(\lambda y \frac{\partial g_{n}}{\partial y}+x \frac{\partial g_{n}}{\partial x}\right)=0
$$

From here and if $\lambda \neq 1$ we get that

$$
g_{n}=e^{\alpha_{0} x /((1-\lambda) y)} x^{(2 \lambda-1) m} G\left(x^{-\lambda} y\right)
$$

where $G$ is an arbitrary function in the variable $x^{-\lambda} y$. If $\lambda=1$ then

$$
g_{n}=x^{m+\alpha_{0} x / y} G\left(\frac{y}{x}\right)
$$

again $G$ is an arbitrary function in the variable $y / x$. Since $g_{n}$ is a polynomial of degree $n-2 m$ we have that $\alpha_{0}=0$. Since the cofactor of $h$ cannot be zero, we have that $\alpha_{1}=-m \neq 0$. So $m>0$. Note that once $\alpha_{0}=0$ the expression (8) with $\lambda=1$ coincides with the expression (9). In order that $g_{n}$ be a polynomial we need that $G(s)=s^{r}$ with $r$ some non-negative integer. 
Therefore, since the degree of $g_{n}$ is $n-2 m$ we have

$$
(2 \lambda-1) m+r-\lambda r=n-2 m, \quad \text { or equivalently } \quad \lambda=\frac{n-m-r}{2 m-r} .
$$

Thus $2 m-r \neq 0$. Then

$$
h_{n}=\left(x z+\frac{m-n+r}{2 m-r} y^{2}\right)^{m} x^{n-2 m-r} y^{r}
$$

with $n \geq 2 m+r$ and

$$
\begin{aligned}
h_{n-1}= & \left(x z+\frac{m-n+r}{2 m-r} y^{2}\right)^{m}\left[2(2 m-r) r(m-n+r) x y^{2} z+\right. \\
& r(r-2 m)^{2} x^{2} z^{2}+2(2 m-r) r(m-n+r) x y^{2} z+g_{n-1}+ \\
& \left.\frac{(m-n+r)\left(4 m^{2}+(2 m+r)(r-n)\right) x^{n-2 m-r-2} y^{r+3}}{(2 m-r)\left((m-n+r) y^{2}+(2 m-r) x z\right)}\right],
\end{aligned}
$$

where $g_{n-1}$ is an arbitrary homogeneous polynomial of degree $n-2 m$.

Computing the terms of degree $n-1$ in (7) we get

$y \frac{\partial h_{n-1}}{\partial x}+z \frac{\partial h_{n-1}}{\partial y}-\left(x z+\frac{m-n+r}{2 m-r} y^{2}\right) \frac{\partial h_{n-2}}{\partial z}+\frac{m-n+r}{2 m-r} \frac{\partial h_{n}}{\partial z}+m x h_{n-2}=0$.

Clearly

$$
f(x, y, z)=y \frac{\partial h_{n-1}}{\partial x}+z \frac{\partial h_{n-1}}{\partial y}+\frac{m-n+r}{2 m-r} \frac{\partial h_{n}}{\partial z}
$$

is a homogeneous polynomial of degree $n-1$. Solving the ordinary differential equation

$$
-\left(x z+\frac{m-n+r}{2 m-r} y^{2}\right) \frac{\partial h_{n-2}}{\partial z}+m x h_{n-2}+f(x, y, z)=0
$$


ON THE DARBOUX INTEGRABILITY OF BLASIUS AND FALKNER-SKAN EQUATIOIS we obtain that

$$
\begin{aligned}
h_{n-2}(x, y, z)= & {\left[(m-n+r) y^{2}+(2 m-r) x z\right]^{m}[K+(2 m-r)} \\
& \left.\int\left[(m-n+r) y^{2}+(2 m-r) x z\right]^{-m-1} f(x, y, z) d z\right],
\end{aligned}
$$

where $K$ is a constant of integration. Since the integrals

$\left[(m-n+r) y^{2}+(2 m-r) x z\right]^{m} \int\left[(m-n+r) y^{2}+(2 m-r) x z\right]^{-m-1} z^{k} d z$

for $k=0,1, \ldots$ are rational functions of the form

$$
\frac{p_{2 k}(x, y, z)}{x^{k+1}}=\frac{-k !(m-n+r)^{k} y^{2 k}+\cdots}{C_{k} x^{k+1}}
$$

where $p_{2 k}(x, y, z)$ is a homogeneous polynomial of degree $2 k$ coprime with $x$ and $C_{k}$ is a constant. Recall that $m-n+r \neq 0$ because $\lambda \neq 0$. Therefore $h_{n-2}(x, y, z)$ can never be a polynomial. So there are no Darboux polynomials $h(x, y, z)$ of degree $n>2$.

For a proof of the next proposition see [4].

Proposition 5. The following statements hold.

(a) If $E=e^{g / h}$ is an exponential factor for the polynomial system (2) and $h$ is not a constant polynomial, then $h=0$ is an invariant algebraic curve. 
(b) Eventually $e^{g}$ can be an exponential factor, coming from the multiplicity of the infinite invariant straight line.

Proposition 6. The unique exponential factors $E$ of system (2) are $e^{x}$ and $e^{y}$, except if $\lambda=-1$ then we have the additional exponential factor $e^{z+x y}$.

Proof. First we prove the proposition for the Blasius system, i.e. for (2) with $\lambda=0$. It follows from Propositions 5 and $4(\mathrm{a})$ that we can write $E=e^{g / z^{n}}$ with $n$ being a non-negative integer. Clearly, after simplifying by $z^{n}$ equation (4), $g$ satisfies

$$
y \frac{\partial g}{\partial x}+z \frac{\partial g}{\partial y}-x z \frac{\partial g}{\partial z}+n x g=\left(\beta_{0}+\beta_{1} x+\beta_{2} y+\beta_{3} z\right) z^{n}
$$

with $\beta_{i} \in \mathbb{C}$ for $i=0,1,2,3$ not all zero, otherwise $g$ would be a Darboux polynomial coprime with $z$ for the Blasius system, and by Proposition 4(a) we know that the Blasius system has no such Darboux polynomials.

Denoting $\bar{g}=\bar{g}(x, y)=g(x, y, 0)$ we get that $\bar{g} \neq 0$ (otherwise $g$ and $z^{n}$ would not be coprime) satisfies (10) restricted to $z=0$, i.e. it satisfies

$$
y \frac{\partial \bar{g}}{\partial x}+n x \bar{g}=0
$$

Solving this linear equation we obtain $\bar{g}=e^{-n x^{2} /(2 y)} g_{1}(y)$, where $g_{1}$ is a function in $y$. Since $\bar{g}$ must be a polynomial we get that $n=0$. Therefore 

we have that $E=e^{g}$ and it satisfies

$$
y \frac{\partial g}{\partial x}+z \frac{\partial g}{\partial y}-x z \frac{\partial g}{\partial z}=\beta_{0}+\beta_{1} x+\beta_{2} y+\beta_{3} z
$$

We consider $F=E e^{-\beta_{2} x-\beta_{3} y}$. Then we can write $F=e^{h}$ with $h=g-$ $\beta_{2} x-\beta_{3} y \in \mathbb{C}[x, y, z]$ that satisfies

$$
y \frac{\partial h}{\partial x}+z \frac{\partial h}{\partial y}-x z \frac{\partial h}{\partial z}=\beta_{0}+\beta_{1} x
$$

Restricting (11) to $z=0$ and setting $\bar{h}=h(x, y, 0)$ we get that

$$
y \frac{\partial \bar{h}}{\partial x}=\beta_{0}+\beta_{1} x
$$

and hence

$$
\bar{h}=f(y)+\frac{\beta_{0} x}{y}+\frac{\beta_{1} x^{2}}{2 y},
$$

where $f(y)$ is a function in the variable $y$. Since $\bar{h}$ is a polynomial we obtain that $\beta_{0}=\beta_{1}=0$. This implies from (11) that $h$ must be zero or a polynomial first integral of the Blasius system. From Proposition 2 this last case is not possible, then $h=0$. Therefore $E=e^{\beta_{2} x+\beta_{3} y}$. This concludes the proof of the proposition for the Blasius system.

Now we consider the Falkner-Skan system. It follows from Proposition 5 that we can write $E=e^{g}$ and from (4) $g$ satisfies

$$
y \frac{\partial g}{\partial x}+z \frac{\partial g}{\partial y}+\left[-x z-\lambda\left(1-y^{2}\right)\right] \frac{\partial g}{\partial z}=\beta_{0}+\beta_{1} x+\beta_{2} y+\beta_{3} z
$$


with $\beta_{i} \in \mathbb{C}, i=0,1,2,3$ not all zero, otherwise $g$ would be a polynomial first integral of the Falkner-Skan system, and by Proposition 2 such first integrals do not exist.

We consider $F=E e^{-\beta_{2} x-\beta_{3} y}$. Then we can write $F=e^{h}$ with $h=$ $g-\beta_{2} x-\beta_{3} y \in \mathbb{C}[x, y, z]$ that satisfies

$$
y \frac{\partial h}{\partial x}+z \frac{\partial h}{\partial y}+\left[-x z-\lambda\left(1-y^{2}\right)\right] \frac{\partial h}{\partial z}=\beta_{0}+\beta_{1} x
$$

We write $h=\sum_{j=0}^{n} h_{j}$ where each $h_{j}=h_{j}(x, y, z)$ is a homogeneous polynomial of degree $j, h_{n} \neq 0$ and $n \geq 1$.

It is easy to check by direct computations that for $n=1$ equation (12) has no solution, and for $n=2$ it has only one solution $h(x, y, z)=z+x y$ when $\lambda=-1, \beta_{0}=1$ and $\beta_{1}=0$. So in what follows we consider $n>2$.

We get that the terms of degree $n+1$ in (12) satisfy

$$
\left[-x z+\lambda y^{2}\right] \frac{\partial h_{n}}{\partial z}=0
$$

Thus $h_{n}=h_{n}(x, y)$ a homogeneous polynomial of degree $n$ in the variables $(x, y)$. Computing the terms of degree $n$ in $(12)$ we get that

$$
y \frac{\partial h_{n}}{\partial x}+z \frac{\partial h_{n}}{\partial y}+\left[-x z+\lambda y^{2}\right] \frac{\partial h_{n-1}}{\partial z}=0 .
$$


ON THE DARBOUX INTEGRABILITY OF BLASIUS AND FALKNER-SKAN EQUATIOI9 Its solution is

$$
h_{n-1}=\tilde{h}_{n-1}(x, y)+\frac{z}{x} \frac{\partial h_{n}}{\partial y}+\frac{y}{x^{2}} \log \left(x z-\lambda y^{2}\right)\left[\lambda y \frac{\partial h_{n}}{\partial y}+x \frac{\partial h_{n}}{\partial x}\right]
$$

where $\tilde{h}_{n-1}=\tilde{h}_{n-1}(x, y)$ is an arbitrary function in the variables $x$ and $y$. Since $h_{n-1}$ is a polynomial we obtain

$$
\lambda y \frac{\partial h_{n}}{\partial y}+x \frac{\partial h_{n}}{\partial x}=0
$$

and hence $h_{n}=h_{n}\left(x^{-\lambda} y\right)$. Since $h_{n} \neq 0$ is a homogeneous polynomial of degree $n>1$, we have $\lambda=-p / q$ with $p$ and $q$ integers such that $p \geq 1$, $q \geq 1, p+q=n$ and $h_{n}=\alpha_{n} x^{p} y^{q}$ where $\alpha_{n} \in \mathbb{C} \backslash\{0\}$. Of course $p \geq 1$ because $\lambda \neq 0$. In short we have that $h_{n}=\alpha_{n} x^{p} y^{q}$. Therefore

$$
h_{n-1}=\tilde{h}_{n-1}(x, y)+\alpha_{n} q x^{p-1} y^{q-1} z .
$$

Computing the terms of degree $n-1$ in (12) we get that

$$
y \frac{\partial h_{n-1}}{\partial x}+z \frac{\partial h_{n-1}}{\partial y}+\left[-x z+\lambda y^{2}\right] \frac{\partial h_{n-2}}{\partial z}=0
$$

that is

$$
\begin{aligned}
h_{n-2}= & \tilde{h}_{n-2}(x, y)+\frac{1}{2} \alpha_{n}\left[2(p-q) y^{2}+(q-1) q x z\right] x^{p-3} y^{q-2} z+ \\
& \frac{z}{x} \frac{\partial \tilde{h}_{n-1}}{\partial y}+\frac{y}{q x^{4}} \log \left(p y^{2}+q x z\right) A,
\end{aligned}
$$


where

$$
A=-\alpha_{n} p(p-q) x^{p} y^{q+1}+q x^{3} \frac{\partial \tilde{h}_{n-1}}{\partial x}-p x^{2} y \frac{\partial \tilde{h}_{n-1}}{\partial y}
$$

Since $h_{n-2}$ must be a homogeneous polynomial of degree $n-2$ we must have that $A=0$. Solving this linear partial differential equation we get that

$$
\tilde{h}_{n-1}(x, y, z)=-\frac{1}{p+2 q} \alpha_{n} p(p-q) x^{p-2} y^{q+1}+G\left(x^{p / q} y\right) .
$$

Since $p+q=n$ and $\tilde{h}_{n-1}$ is a homogeneous polynomial of degree $n-1$ we obtain

$$
\tilde{h}_{n-1}(x, y, z)=-\frac{1}{p+2 q} \alpha_{n} p(p-q) x^{p-2} y^{q+1} .
$$

Therefore

$$
\begin{aligned}
h_{n}= & \alpha_{n} x^{p} y^{q}, \\
h_{n-1}= & \alpha_{n} q x^{p-1} y^{q-1} z-\frac{1}{p+2 q} \alpha_{n} p(p-q) x^{p-2} y^{q+1}, \\
h_{n-2}= & \tilde{h}_{n-2}(x, y)+\frac{1}{2} \alpha_{n}\left[2(p-q) y^{2}+(q-1) q x z\right] x^{p-3} y^{q-2} z- \\
& \frac{1}{p+2 q} \alpha_{n} p(p-q)(q+1) x^{p-3} y^{q} z .
\end{aligned}
$$

Note that $h_{n-1}$ is a polynomial if $p \geq 1$ and $q \geq 1$, so $n \geq 2$; and that $h_{n-2}$ is a polynomial if $p \geq 3$ and $q \geq 2$, so $n \geq 5$.

Working in a similar way with the terms of degree $n-2$ in (5) we get that

$$
\begin{aligned}
\tilde{h}_{n-2}= & \frac{\alpha_{n} x^{p-4} y^{q-2}}{2 q(p+2 q)^{2}}\left[-q^{2}(p+2 q)^{2} x^{4}+\right. \\
& \left.p\left(p^{3}(q-1)-6 q^{3}+p q^{2}(8+q)-p^{2} q(1+2 q)\right) y^{4}\right]
\end{aligned}
$$


and

$$
\begin{aligned}
h_{n-3}= & \frac{1}{6(p+2 q)^{2}}\left[6(p+2 q)^{2} \tilde{h}_{n-3}(x, y)+\alpha_{n} x^{p-5} y^{q-3} z\left(3(2-q) q(p+2 q)^{2} x^{4}+\right.\right. \\
& 3(p-4)(p-q)\left(6 q^{2}-p^{2}-2 p q+p^{2} q-p q^{2}\right) y^{4}+ \\
& 3(-p+q)(p+2 q)\left(p+2 q-p q-4 q^{2}+p q^{2}\right) x y^{2} z+ \\
& \left.\left.(q-2)(q-1) q(p+2 q)^{2} x^{2} z^{2}\right)\right]
\end{aligned}
$$

where $\tilde{h}_{n-3}$ is an arbitrary polynomial of degree $n-3$. Since $h_{n-3}$ is a polynomial we have that $p \geq 5$ and $q \geq 3$, so $n \geq 8$.

In short if there exists an exponential factor $e^{h}$ satisfying (12), with $h$ a polynomial of degree $n$, then $h_{n-s}$ is a polynomial if $p \geq 2 s-1$ and $q \geq s$, so $n \geq 3 s-1$. Thus for $s=n-1$ we get that $h_{1}$ is a polynomial if $n \geq 3(n-1)-1=3 n-4$, i.e. if and only if $4 \geq 2 n$, or equivalently if $n=1,2$. But as we have mentioned by direct computations the unique exponential factors $e^{h}$ with $h$ of degree 1 and 2 are the ones described in the statement of the proposition. This completes the proof of the proposition.

Finally the last statement of Theorem 1 follows from the next result.

Proposition 7. The Falkner-Skan system and Blasius system have no first integrals of Darboux type. 
Proof. Let $H=H(x, y, z)$ be a first integral of system (2), then it must satisfy that

$$
y \frac{\partial H}{\partial x}+z \frac{\partial H}{\partial y}-\left[x z+\lambda\left(1-y^{2}\right)\right] \frac{\partial H}{\partial z}=0 .
$$

Assume $\lambda=0$. Then by Propositions 4 and 5 the only one Darboux polynomial of system (2) is $z$ and its only exponential factors are $e^{x}$ and $e^{y}$. So if system (2) has a Darboux first integral it must be of the form $H=z^{a} e^{b_{1} x+b_{2} y}$. Thus equation (13) becomes

$$
z^{a} e^{b_{1} x+b_{2} y}\left(-a x+b_{1} y+b_{2} z\right)=0
$$

or equivalently

$$
-a x+b_{1} y+b_{2} z=0
$$

Assume $\lambda=1 / 2$. Then by Propositions 4 and 5 the only Darboux polynomial of system $(2)$ is $1-y^{2}+2 x z$ and its only exponential factors are $e^{x}$ and $e^{y}$. So if system (2) has a Darboux first integral it must be of the form $H=\left(1-y^{2}+2 x z\right)^{a} e^{b_{1} x+b_{2} y}$, and equation (13) becomes

$$
\left(1-y^{2}+2 x z\right)^{a} e^{b_{1} x+b_{2} y}\left(-a x+b_{1} y+b_{2} z\right)=0
$$


ON THE DARBOUX INTEGRABILITY OF BLASIUS AND FALKNER-SKAN EQUATIOAS or equivalently

$$
-a x+b_{1} y+b_{2} z=0 .
$$

Assume $\lambda=-1$. Then by Propositions 4 and 5 system (2) does not have Darboux polynomials and its only exponential factors are $e^{x}, e^{y}$ and $e^{z+x y}$. So if system (2) has a Darboux first integral it must be of the form $H=e^{b_{1} x+b_{2} y+b_{3}(z+x y)}$, and equation (13) becomes

$$
e^{b_{1} x+b_{2} y+b_{3}(x y+z)}\left(b_{3}+b_{1} y+b_{2} z\right)=0,
$$

or equivalently

$$
b_{3}+b_{1} y+b_{2} z=0 .
$$

Finally suppose that $\lambda \notin\{-1,0,1 / 2\}$. Then by Propositions 4 and 5 system (2) has no Darboux polynomials and only has the exponential factors $e^{x}$ and $e^{y}$. So if system (2) has a Darboux first integral it must be of the form $H=e^{b_{1} x+b_{2} y}$, and equation (13) becomes

$$
e^{b_{1} x+b_{2} y}\left(b_{1} y+b_{2} z\right)=0,
$$

or equivalently

$$
b_{1} y+b_{2} z=0 \text {. }
$$


From equations (13)-(17) it follows that $a=0$ and $b_{k}=0$ for $k=1,2,3$ when these coefficients appears in some of the candidates to have a Darboux first integral. Hence system (2) does not admit a Darboux first integral.

\section{ACKNOWLEDGEMENTS}

We thank Adam Mahdi for helping us to write this manuscript in its present form.

The first author is supported by the grants MCYT/FEDER MTM 200803437 and Generalitat de Catalunya 2009SGR410, and ICREA Academia and FP7-PEOPLE-2012-IRSES-316338 and 318999. The second author is partially supported by FCT through CAMGDS, Lisbon.

\section{REFERENCES}

[1] D.J. Acheson, Elementary fluid dynamics, Oxford Applied Mathematics and Computing Science Series, The Clarendon Press Oxford University Press, New York, 1990.

[2] H. Blasius, Grenzschichten in flussigkeiten mit kleiner reibung, Z. Math. Phys. 56 (1908), 1-37.

[3] B. Brighi, The equation $f^{\prime \prime \prime}+f f^{\prime \prime}+g\left(f^{\prime}\right)=0$ and the associated boundary value problems, Results Math. 61 (2012), 355-391.

[4] C. Christopher, J. Llibre, and J.V. Pereira, Multiplicity of invariant algebraic curves in polynomial vector fields, Pacific J. Math. 229 (2007), 63-117.

[5] W.A. Coppel, On a differential equation of boundary-layer theory, Philos. Trans. Roy. Soc. London. Ser. A 253 (1960), 101-136.

[6] G. Falkner and S.W. Skan, Solutions of the boundary layer equations., Phil. Mag. 7 (1931), 865-896. 
ON THE DARBOUX INTEGRABILITY OF BLASIUS AND FALKNER-SKAN EQUATIO2S

[7] S. P. Hastings and W. Troy, Oscillatory solutions of the Falkner-Skan equation, Proc. Roy. Soc. London Ser. A 397 (1985), no. 1813, 415-418.

[8] S. P. Hastings and W. C. Troy, Oscillating solutions of the Falkner-Skan equation for negative $\beta$, SIAM J. Math. Anal. 18 (1987), no. 2, 422-429.

[9] S.P. Hastings and W.C. Troy, Oscillating solutions of the Falkner-Skan equation for positive $\beta$, J. Differential Equations 71 (1988), no. 1, 123-144.

[10] T. Hayat, M. Hussain, S. Nadeem and S. Mesloub, Falkner-Skan wedge flow of a power-law fluid with mixed convection and porous medium, Comput. \& Fluids 49 (2011), 22-28.

[11] K. Hsiao and C. Hsu, Conjugate heat transfer of mixed convection for visco-elastic fluid past a triangular fin, Nonlinear Anal. Real World Appl. 10 (2009), 130-143.

[12] J. Llibre, Integrability of polynoial differential systems, Handbook of Differential Equations, Ordinary Differential Equations, Eds. A. Cañada, P. Drabek and A. Fonda, 1 (2004), 437-533.

[13] J. Llibre and M. Messias, Large amplitude oscillations for a class of symmetric polynomial differential systems in $\mathbb{R}^{3}$, Annals of the Brazilian Academy of Sciences $\mathbf{7 9}$ (2007), 1-13.

[14] J. Llibre and X. Zhang, Darboux theory of integrability in $c^{n}$ taking into account the multiplicity, J. of Differential Equations 246 (2009), 541-551.

[15] O. Padé, On the solution of Falkner-Skan equations, J. Math. Anal. Appl. 285 (2003), $264-274$

[16] L. Rosenhead, Laminar boundary layers, Clarendon Press, Oxford, 1963.

[17] C. Sparrow and H.P.F. Swinnerton-Dyer, The Falkner-Skan equation. II. Dynamics and the bifurcations of $P$ - and Q-orbits, J. Differential Equations 183 (2002), no. 1, $1-55$.

[18] H.P.F. Swinnerton-Dyer and C.T. Sparrow, The Falkner-Skan equation. I. The creation of strange invariant sets, J. Differential Equations 119 (1995), no. 2, 336-394. 
[19] H. Weyl, On the differential equations of the simplest boundary-layer problem, Ann. Math. 43 (1942), 381-407.

${ }^{1}$ Departament de Matemàtiques, Universitat Autònoma de Barcelona, 08193 Bellaterra, Barcelona, Catalonia, Spain

E-mail address: jllibre@mat.uab.cat

4 Departamento de Matemática, Instituto Superior Técnico, Av. Rovisco Pais 1049-001, Lisboa, Portugal

E-mail address: cvalls@math.ist.utl.pt 